\title{
Piperlongumine induces apoptosis and reduces bortezomib resistance by inhibiting STAT3 in multiple myeloma cells
}

\author{
Yao Yao ${ }^{1,2,4, *}$, Yueyue Sun ${ }^{1,2,4, *}$, Min Shi ${ }^{1,2,4, *}$, Dandan Xia ${ }^{1,2,4}$, Kai Zhao ${ }^{1,2,4}$, Lingyu \\ Zeng ${ }^{1,2,4}$, Ruosi Yao $^{1,2,4}$, Ying Zhang ${ }^{3}$, Zhenyu $\mathrm{Li}^{2}$, Mingshan Niu ${ }^{1,2,4}$, Kailin $\mathrm{Xu}^{1,2,4}$ \\ ${ }^{1}$ Blood Diseases Institute, Xuzhou Medical College, Xuzhou, Jiangsu, China \\ ${ }^{2}$ Department of Hematology, The Affiliated Hospital of Xuzhou Medical College, Xuzhou, Jiangsu, China \\ ${ }^{3}$ Laboratory of Pathology, Xuzhou Medical College, Xuzhou, Jiangsu, China \\ ${ }^{4}$ Key Laboratory of Bone Marrow Stem Cell, Jiangsu Province, Xuzhou, China \\ *These authors have contributed equally to this work \\ Correspondence to: Kailin XU, email: lihmd@163.com, kailinxu_Xzmc@126.com \\ Mingshan Niv, email: msniu24@126.com \\ Keywords: multiple myeloma, STAT3, piperlongumine, bortezomib resistance, immunoproteasome \\ Received: September 17, $2015 \quad$ Accepted: September 02, $2016 \quad$ Published: September 13, 2016
}

\section{ABSTRACT}

Effective new therapies are urgently needed for the treatment of multiple myeloma (MM), an incurable hematological malignancy. In this study, we evaluated the effects of piperlongumine on MM cell proliferation both in vivo and in vitro. Piperlongumine inhibited the proliferation of MM cells by inducing cell apoptosis and blocking osteoclastogenesis. Notably, piperlongumine also reduced bortezomib resistance in MM cells. In a disseminated MM mouse model, piperlongumine prolonged the survival of tumor-bearing mice without causing any obvious toxicity. Mechanistically, piperlongumine inhibited the STAT3 signal pathway in MM cells by binding directly to the STAT3 Cys712 residue. These findings suggest that the clinical use of piperlongumine to overcome bortezomib resistance in MM should be evaluated.

\section{INTRODUCTION}

Multiple myeloma (MM), a plasma cell disorder characterized by anemia, lytic bone disease, renal disease, and immune dysfunction, is the second most common hematologic malignancy $[1,2]$. Many novel therapies, including proteasome inhibitors and immunomodulatory agents (IMiDs), have improved MM treatment during the past decade. The first-in-class proteasome inhibitor bortezomib is commonly used to treat MM. However, relapse following bortezomib treatment remains inevitable, and novel targets are needed for MM therapy [3-6].

Piperlongumine is an alkaloid isolated from the long pepper (Piper longum L.) that possesses antiinflammatory, anti-platelet aggregation, and anti-tumor properties [7-9]. Piperlongumine exerts anti-tumor effects through a variety of pathways, including the induction of reactive oxygen species (ROS) accumulation, activation of C/EBP homologous protein (CHOP), suppression of LMP-1 (EBV-encoded oncogene) expression, activation of AMPK phosphorylation, inhibition of NF- $\mathrm{KB}$, and promotion of autophagy [10-17]. Recently, Jarvius et al. found that piperlongumine inhibited the function of the ubiquitin-proteasome system (UPS) and subsequent ROS generation [18]. However, the mechanisms underlying these effects remain unclear.

Here, we investigated whether piperlongumine has anti-tumor activity in MM cells. We found that piperlongumine induced apoptosis not only in BTZ-sensitive, but also in BTZ-resistant, MM cells. Furthermore, we report for the first time that piperlongumine inhibited the activation of STAT3 by directly binding to Cys 712 near the $\mathrm{SH} 2$ domain; piperlongumine had no anti-myeloma effects in cells with mutant STAT3 (C712A).

\section{RESULTS}

Piperlongumine inhibits proliferation and induces apoptosis in MM cells

The effects of piperlongumine on the growth of various $\mathrm{MM}$ cell lines, including cells either sensitive or resistant to bortezomib (BTZ), were first determined by 
CCK-8 assay. Incubation with piperlongumine for $48 \mathrm{~h}$ inhibited MM cell growth in a dose-dependent manner, with $\mathrm{IC}_{50}$ values ranging from 1 to $5 \mu \mathrm{M}$ (Figure $1 \mathrm{~B}$ and Table 1). Treatment with different concentrations of piperlongumine for 24,48 , or $72 \mathrm{~h}$ also inhibited the growth of NCI-H929 cells in a dose- and time-dependent manner (Figure 1C). Similar results were obtained in IM9 and OPM2 cells (Supplementary Figure S1A); however, HS-5 stromal cells and normal hematopoietic cells were less sensitive to piperlongumine (Supplementary Figure S2).

Cell cycle distribution and apoptosis rates were then examined in MM cells to investigate the mechanisms underlying piperlongumine's effects. Piperlongumine treatment increased the proportion of NCI-H929 cells in the $\mathrm{S}$ phase in a time-dependent manner (Figure 1D). In addition, piperlongumine increased apoptosis in a dose-dependent manner (Figure 1E). Similar cell cycle distribution and apoptosis results were obtained in IM9 and OPM2 cells (Supplementary Figure S1B-S1C).

\section{Piperlongumine induces $\mathrm{MM}$ cell apoptosis through both Fas- and mitochondria-dependent pathways}

To determine how piperlongumine blocks cell cycle progression, a BrdU incorporation assay was performed to measure DNA synthesis. DNA synthesis decreased markedly in NCI-H929 cells after exposure to
A
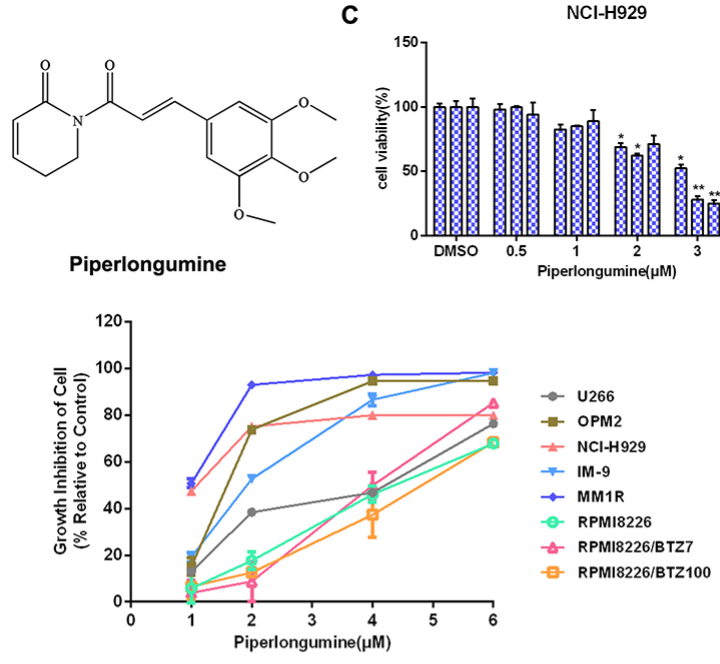

D

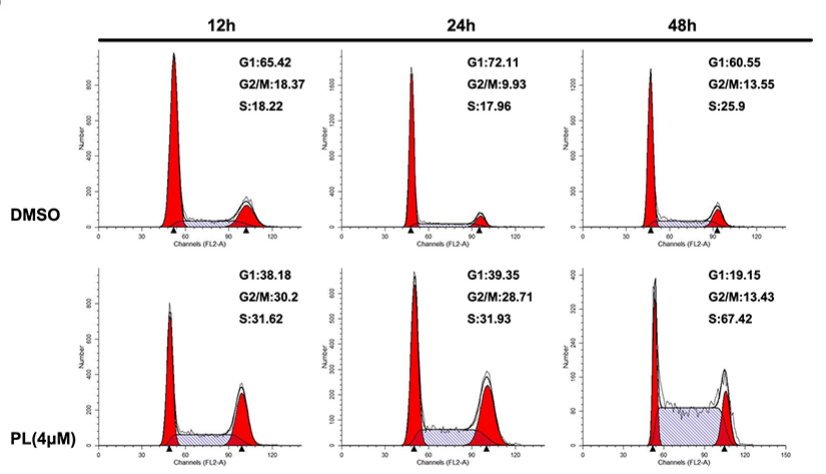

E

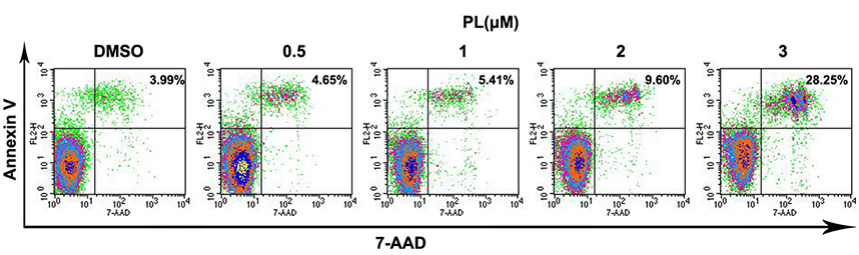

Figure 1: Piperlongumine inhibits cell proliferation and induces apoptosis in MM cells. A. The structure of piperlongumine. B. Eight types of MM cells were treated with different concentrations of piperlongumine for $48 \mathrm{~h}$ and relative cell viabilities were then measured using a CCK-8 assay. C. Cell viability was measured in NCI-H929 cells treated with different doses of piperlongumine for 24, 48 , or $72 \mathrm{~h}$. D. After NCI-H929 cells were treated with $4 \mu \mathrm{M}$ piperlongumine for 12, 24, or $48 \mathrm{~h}$, relative numbers of cells in each cell cycle phase were analyzed by flow cytometry. E. NCI-H929 cells were treated with different concentrations of piperlongumine for $48 \mathrm{~h}$ and apoptosis rates were determined. All CCK-8 assay results were obtained from three independent experiments. 
Table 1: The IC50 values of seven human MM cell lines on $48 \mathrm{~h}$

\begin{tabular}{lc}
\hline Tumor cell line & IC50 ( $1 \mathbf{M})$ \\
\hline OPM2 & $2.7 \pm 0.5$ \\
MM1R & $0.9 \pm 0.2$ \\
RPMI8226 & $2.8 \pm 0.8$ \\
NCI-H929 & $1.3 \pm 0.4$ \\
IM-9 & $2.1 \pm 0.2$ \\
U266 & $5.7 \pm 0.9$ \\
RPMI8226/BTZ7 & $3.3 \pm 0.8$ \\
RPMI8226/BTZ100 & $3.3 \pm 0.9$ \\
\hline
\end{tabular}

piperlongumine (Figure 2A). As expected, the expression of cyclin $\mathrm{A}$, which promotes progression from the $\mathrm{S}$ to the G2 phase, decreased, while cyclin E expression increased; cyclin-dependent kinase (CDK) expression did not change (Figure 2B). Moreover, piperlongumine increased apoptosis in both time- and dose-dependent manners, as measured by caspase family (caspase-3, -9, or -8) cleavage and activity. As shown in Figure $2 \mathrm{C}$ and $2 \mathrm{D}$, marked induction of caspase- $3,-9$, or -8 activity and cleavage were observed. Similar results were obtained in OPM2 and IM9 cells (Supplementary Figure S1D). Piperlongumine also decreased levels of the anti-apoptotic protein Bcl-2 and increased the Bax/Bcl-2 ratio in NCI-H929 cells (Figure 2E). In addition, piperlongumine treatment disrupted the mitochondrial membrane potential, as revealed by an increase in green fluorescence resulting from the cytosolic accumulation of monomeric JC-1 (Figure 2F). Intracellular ROS levels also increased after treatment (Supplementary Figure S1E). These data suggest that piperlongumine induces MM cell apoptosis through both Fas- and mitochondria-dependent pathways.

\section{Piperlongumine blocks osteoclastogenesis and cytokine secretion}

Proliferation, survival, and avoidance of immune surveillance in MM cells all depend on the bone marrow (BM) microenvironment [19-21]. We therefore investigated the effects of piperlongumine on the BM microenvironment by measuring the secretion of VEGF from $\mathrm{MM}$ and BM stem cells, as well as osteoclast formation. As shown in Figure 3A, VEGF secretion decreased in NCI-H929 MM cells after piperlongumine treatment alone or together with co-cultured HS-5 cells (Figure 3A). MM cell growth also decreased after piperlongumine treatment with or without HS-5 cells (Figure 3B). Because osteolytic bone disease results from excessive osteoclast activation in most patients [22], an osteoclast formation assay was performed. As shown in Figure 3C, piperlongumine decreased numbers of TRACPpositive multinuclear cells in a dose-dependent manner.
Together, these results indicate that piperlongumine may also inhibit MM cell growth and survival by altering the BM microenvironment.

\section{Piperlongumine inhibits the STAT3 signaling pathway in MM cells}

To identify signal transduction pathways involved in the effects of piperlongumine, we measured activation of the NF- $\mathrm{B}$, MAPK, PI3K/AKT, UPS, and JAK/ STAT3 pathways. Piperlongumine inhibited UPS function in NCI-H929 cells as demonstrated by the increased accumulation of poly-ubiquitinated proteins (Figure 4A). Furthermore, piperlongumine markedly inhibited STAT3 activity (Figure 4B), but did not affect the phosphorylation of JAK2, which acts upstream of STAT3 (Figure 4C). Next, we measured the levels of molecules downstream of STAT3, including c-myc, p21, p27, and survivin. Piperlongumine decreased survivin and c-myc levels and increased p21 and p27 levels (Figure 4D-4F). Activation of the NF- $\kappa \mathrm{B}$ and MAPK signal pathways were not affected by piperlongumine, and PI3K/AKT activation was low in most MM cells (MM1R and JJN3) without cytokine treatment (IGF) (Supplementary Figure S3).

\section{Piperlongumine binds directly to STAT3 and inhibits its activity}

To explore whether piperlongumine targets STAT3 directly, we performed a STAT3 pull-down assay using biotinylated piperlongumine. Treatment with biotin-piperlongumine resulted in STAT3 pulldown in 293T cell lysates, and treatment with unlabeled piperlongumine attenuated this binding (Figure 4G). This result and previous findings [23] indicate that piperlongumine may inhibit STAT3 activity by forming a covalent linkage with a specific cysteine residue. To confirm this, we mutated Cys712 to Ala; this mutation greatly reduced pull-down of STAT3 with biotin-piperlongumine (Figure $4 \mathrm{G}$ and $4 \mathrm{H}$ ). We then developed cell lines that stably expressed either 
exogenous constitutively-active STAT3 (STAT3-CA) or C712A mutant STAT3As shown in Figure 4I, piperlongumine treatment inhibited growth in cells expressing vector or STAT3-CA; however, the antiproliferative effects of piperlongumine were reduced in the cells expressing C712A mutant STAT3. These data suggest that piperlongumine inhibits STAT3 function by binding directly to the Cys 712 residue.

\section{Piperlongumine reduces bortezomib resistance in MM cells}

To explore the ability of piperlongumine to overcome BTZ resistance, we replicated the above experiments in BTZ-resistant cells. Cytotoxicity increased in both BTZ-sensitive and BTZ-resistant cells after exposure to a combination of BTZ and piperlongumine
A

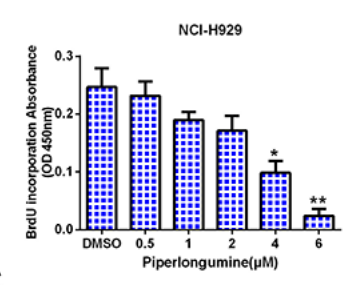

C
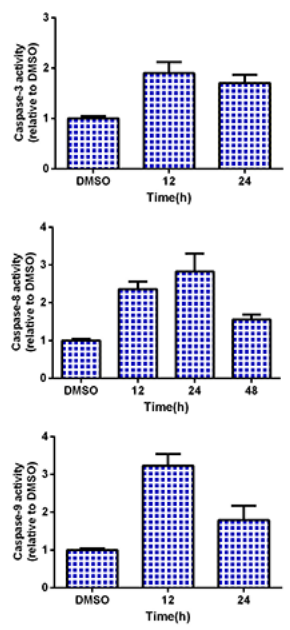

E

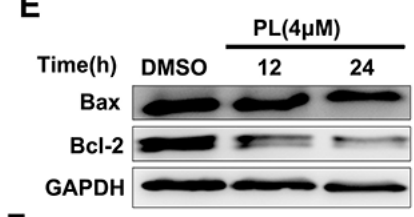

B

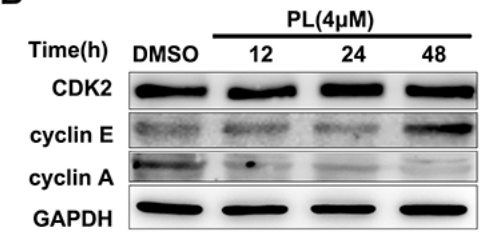

D
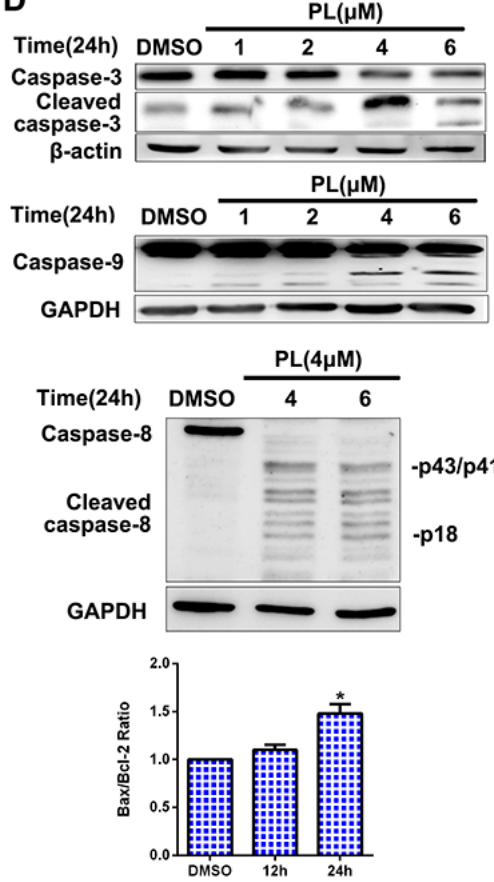

$F$
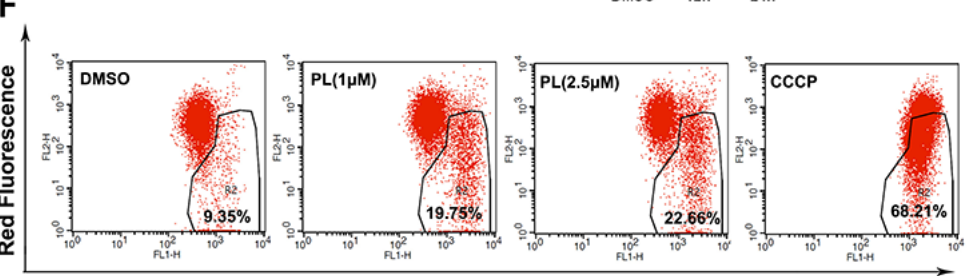

Green Fluorescence

Figure 2: Piperlongumine inhibits DNA synthesis and stimulates apoptosis via Fas- and mitochondria-dependent pathways. A. NCI-H929 cells were treated with piperlongumine $(0.5,1,2,4$, or $6 \mu \mathrm{M})$ for $24 \mathrm{~h}$, and a BrdU incorporation assay was then performed. Data are representative of three independent experiments. ${ }^{*} P<0.05, * * P<0.01$, compared to control. B-C. NCI-H929 cells were treated with $4 \mu \mathrm{M}$ piperlongumine for 12, 24, or $48 \mathrm{~h}$; cyclins and CDK2 levels were then measured, and caspase activity was measured by colorimetric assay. D. NCI-H929 cells were treated with piperlongumine for $24 \mathrm{~h}$, and cleaved caspase-3, caspase-9, and caspase-8 levels were measured. E. NCI-H929 cells were treated with $4 \mu \mathrm{M}$ piperlongumine for 12 or $24 \mathrm{~h}$ and Bcl-2 and Bax levels were measured. Quantitative analysis was performed using Image J software, with normalization to GAPDH expression. F. NCI-H929 cells were treated with 1 or $2.5 \mu \mathrm{M}$ piperlongumine for $12 \mathrm{~h}$; CCCP was used as the positive control. Fluorescence was then measured by flow cytometry. 
$(1.5 \mu \mathrm{M})$ (Figure 5A and 5B). Piperlongumine reduced the viability of BTZ-resistant cells by promoting apoptosis and decreasing STAT3 phosphorylation (Figure 5C and 5D).

Next, we examined the accumulation of polyubiquitinated proteins in BTZ-resistant cells after preexposure to piperlongumine. Treatment with bortezomib or piperlongumine reduced the accumulation of polyubiquitinated proteins. In contrast, pre-exposure to piperlongumine in combination with BTZ greatly increased the accumulation of poly-ubiquitinated proteins (Figure 5E and Supplementary Figure S4). $\beta 5$ i expression was increased, while $\beta 5$ subunit expression was slightly decreased; however, $\beta 1$ and $\beta 1 \mathrm{i}$ (LMP2) levels were unchanged (Figure 5F and Supplementary Figure S4). Proteasome catalytic activities, especially chymotrypsinlike activity, were increased by piperlongumine (Figure $5 \mathrm{G}$ and Supplementary Figure S4) [24]. Taken together, these results suggest that piperlongumine also exerts cytotoxic effects in BTZ-resistant cells by triggering apoptosis and inhibiting the STAT3 signal pathway, and partially restores BTZ sensitivity by increasing $\beta 5$ i levels.

\section{Piperlongumine inhibits human MM cell growth in vivo}

We next examined the in vivo efficacy of piperlongumine using a human MM xenograft mouse model [25]. Mice treated with piperlongumine showed delayed tumor growth $(P<0.01$, Figure 6A). Western blotting analysis of tumors harvested from these mice indicated that cleaved caspase-3 levels increased, and p-STAT3 levels decreased, after piperlongumine treatment (Figure 6B). These results suggest that piperlongumine also exerts antiMM activity in vivo in the plasmacytoma model.

The in vivo efficacy of piperlongumine was further evaluated in a disseminated MM model. Piperlongumine treatment alone markedly reduced tumor growth (Figure 6C and $\mathrm{D}, P<0.01$ ) and survival (Figure 6E) compared to vehicle treatment. The mice in
A

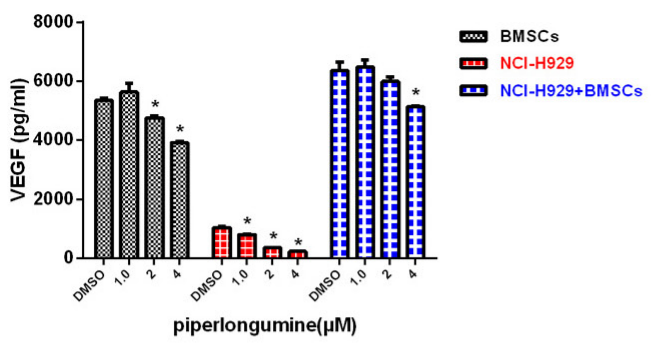

B
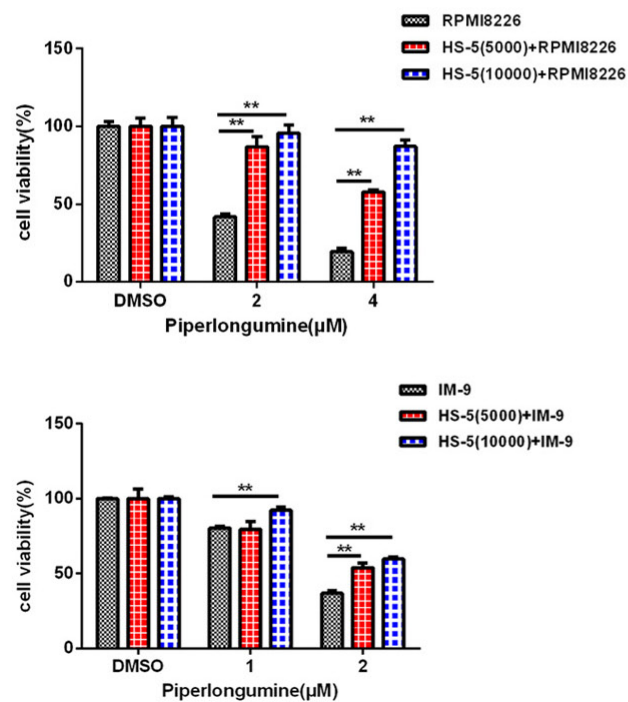

c

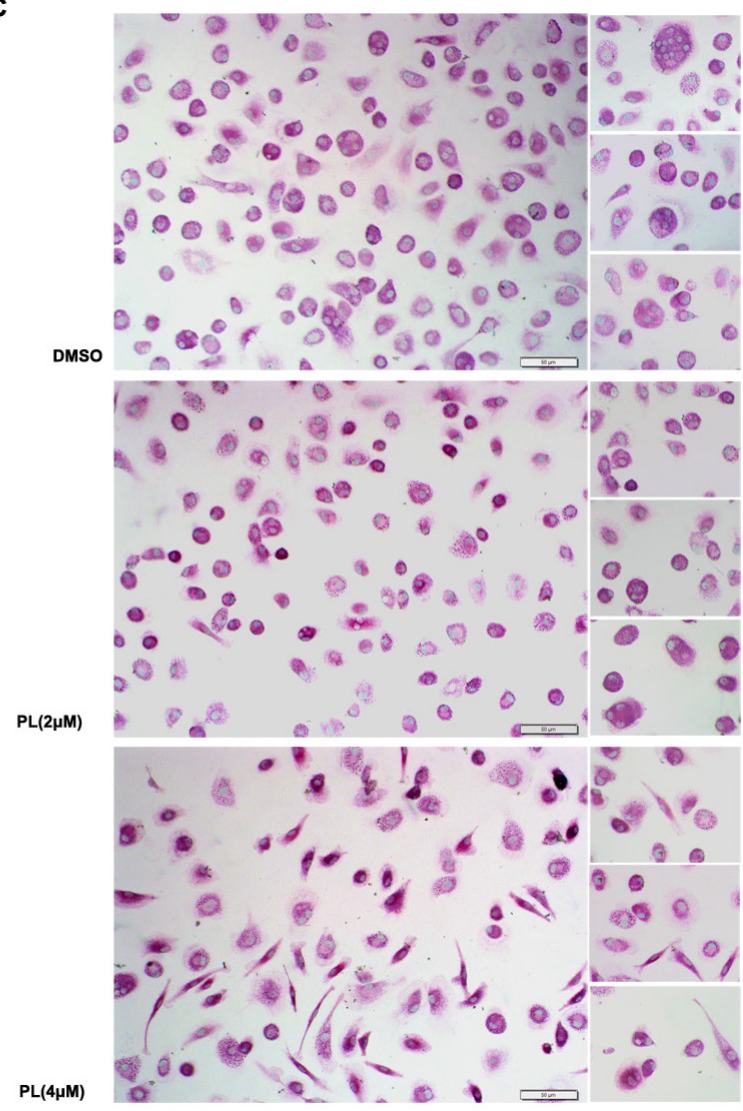

Figure 3: Piperlongumine targeted MM cells in the BM microenvironment and inhibited osteoclast formation. A. NCI-H929 cells, cultured alone or with HS-5 cells, were treated with varying doses of piperlongumine, and conditioned media were collected for the measurement of VEGF levels using an ELISA. B. RPMI8226 or IM-9 cells were seeded into a 96-well microplate alone or with HS-5 cells, then treated with different concentrations of piperlongumine for $48 \mathrm{~h}$ and analyzed with a CCK-8 assay. Results are shown as the mean \pm SD of three independent experiments. C. PBMCs isolated from normal donors $(n=3)$ were incubated with piperlongumine, and the TRACP assay was performed to measure the formation of multinuclear osteoclast cells. The results are representative of three independent experiments. 
all three groups maintained similar body weights during treatment (Figure 6F). H\&E and immunohistochemical staining were used to evaluate pathology in bone marrow isolated from the mice after drug treatment. As shown in Figure 6I, piperlongumine treatment decreased numbers of hemorrhages and infiltrated human myeloma cells compared to the control group. Bone lesions and pro-inflammatory cytokines were also evaluated in the disseminated MM model. Osteoclast (OC) hyperactivation results primarily from increased production of multiple "OC-activating factors" in the
BM microenvironment (e.g., RNAKL, IL-6, IL-3, TNF- $\alpha$, and IL-1 $\beta$ ) $[26,27]$. As shown in Figure $6 \mathrm{G}$, $\mathrm{H}$, and $6 \mathrm{~J}$, IL-6, IL-1 $\beta$, RNAKL, and IL-18 levels decreased after piperlongumine treatment $(P<0.05)$. In contrast, levels of osteoprotegerin (OPG), a decoy receptor for RANKL, increased in the piperlongumine group compared to the control and BTZ groups $(P<0.01)$. Taken together, these data demonstrate that piperlongumine also exerted anti-MM effects and partially prevented bone resorption in a disseminated human MM model.

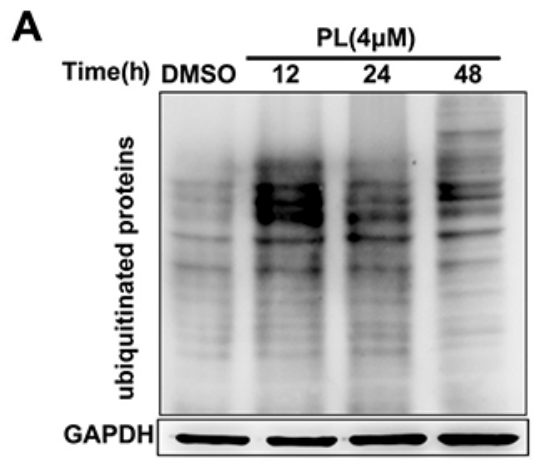

B

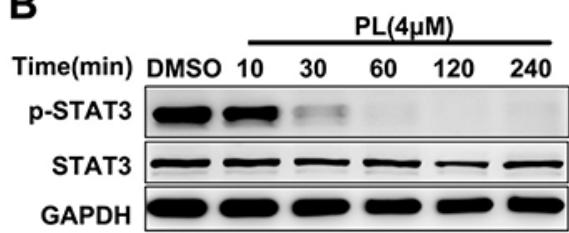

C

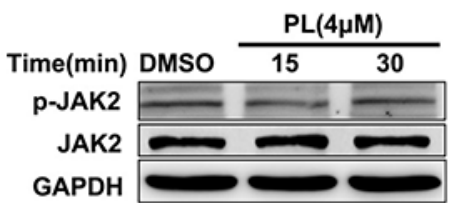

D

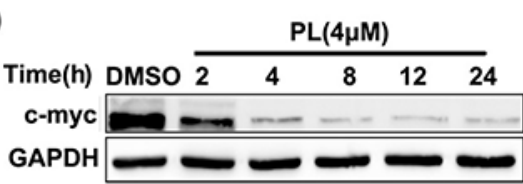

E

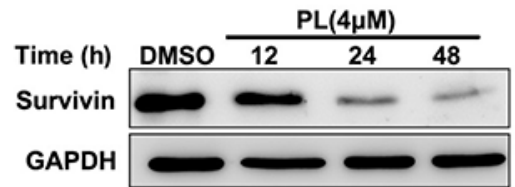

$\mathbf{F}$ $\mathrm{PL}(4 \mu \mathrm{M})$

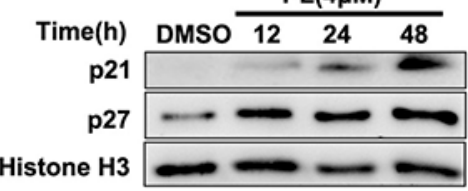

G

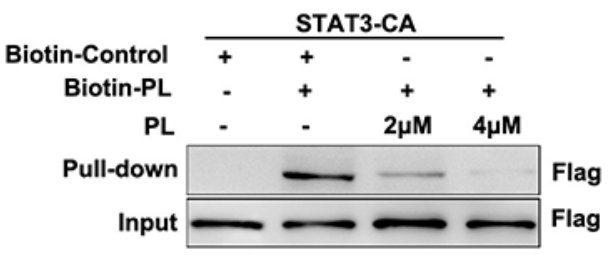

H

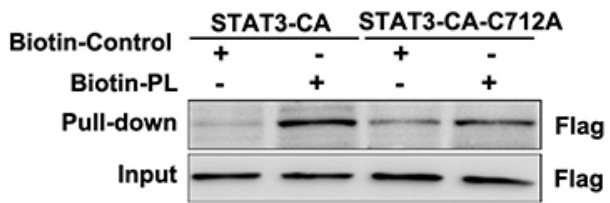

I

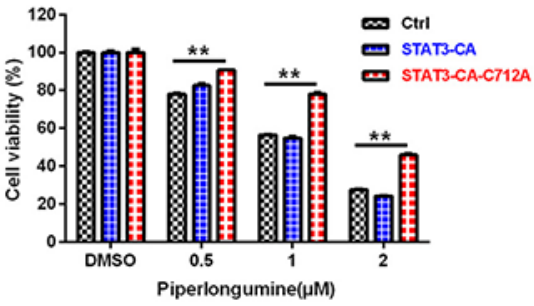

Figure 4: Piperlongumine inhibits STAT3 signal pathway activation. A. NCI-H929 cells were treated with $4 \mu \mathrm{M}$ piperlongumine, and poly-ubiquitinated protein levels were measured. B-C. NCI-H929 cells were treated with $4 \mu \mathrm{M}$ piperlongumine for different amounts of time, and STAT3, p-STAT3, p-JAK2, and JAK2 levels were then measured. D-F. NCI-H929 cells were treated with $4 \mu \mathrm{M}$ piperlongumine for different amounts of time, and c-myc, survivin, p21, and p27 levels were then measured. The data shown are representative of three independent experiments. G. 293 T cells were transiently transfected with Flag-STAT3-CA plasmids and then treated with biotinylated piperlongumine $(20 \mu \mathrm{M})$ for $2 \mathrm{~h}$ in the presence or absence of pretreatment with unlabled piperlongumine for $1 \mathrm{~h}$. The whole cell lysates were subjected to pull-down analysis with the use of streptavidin beads. Captured proteins were analyzed by Western blotting. H. 293T cells were transiently transfected with Flag-STAT3-CA or Flag-STAT3-CA-C712A plasmids and and then treated with biotinylated piperlongumine $(20 \mu \mathrm{M})$ for $2 \mathrm{~h}$. The whole cell lysates were subjected to pull-down analysis with the use of streptavidin beads. Captured proteins were analyzed by Western blotting. I. NCI-H929 cells were infected with vector control, STAT3-CA, or STAT3-CA-C712A expression viruses, then treated with piperlongumine $(0.5,1$, or $2 \mu \mathrm{M})$ for $24 \mathrm{~h}$ and analyzed using a CCK- 8 assay. 


\section{DISCUSSION}

In this study, we investigated the biological mechanisms by which piperlongumine exerts anticancer effects in human myeloma cells. We found that piperlongumine inhibits the proliferation of MM cells both in vitro and in vivo by inducing cell apoptosis. However, sensitivity to piperlongumine varies slightly in different MM cell lines, and especially in RPMI8226 and U266 cells. Consistent with previous reports, both of them are less sensitive to many anti-tumor agents than other MM cell lines $[28,29]$. This may be due to the activity of multiple oncogenic signaling pathways that prevent apoptosis and increase drug resistance in RPMI8226 and U226 cells,
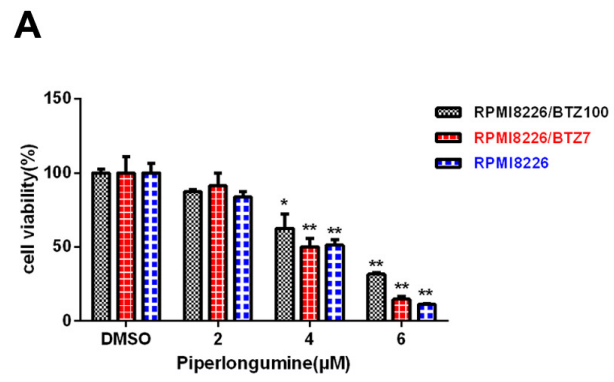

C

\section{B}
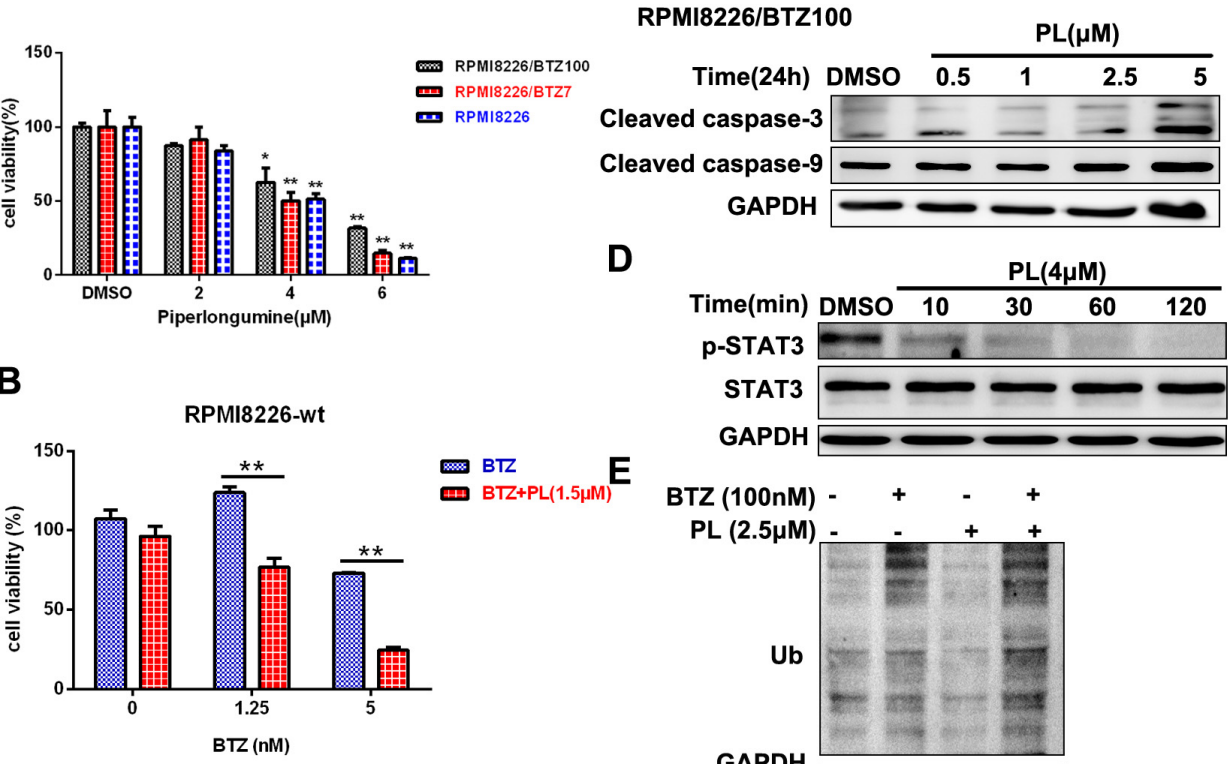

D
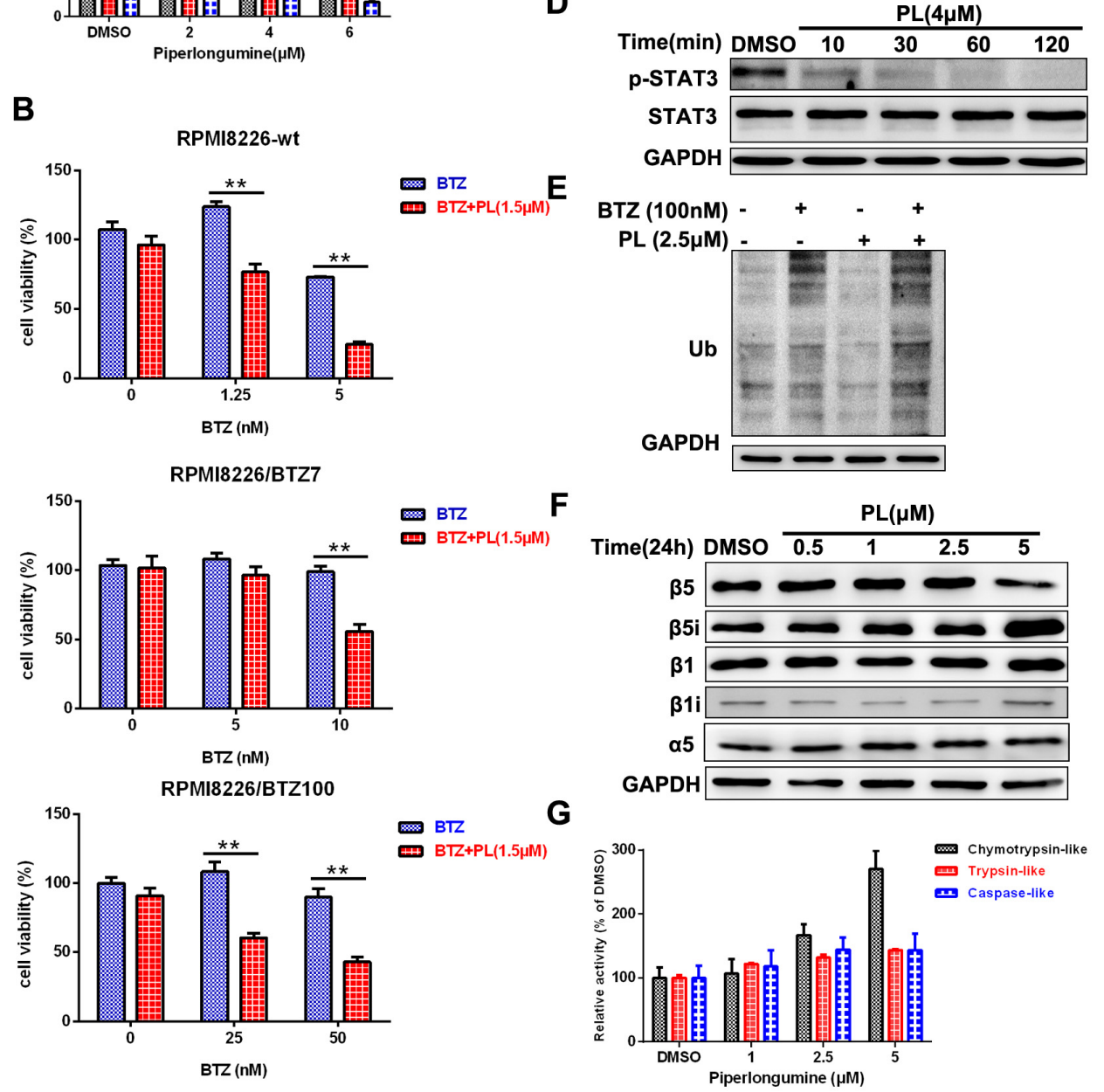

Figure 5: Piperlongumine inhibits viability in BTZ-resistant MM cells. A. RPMI8226, RPMI8226/BTZ7, and RPMI8226/ BTZ100 cells were treated with piperlongumine for $24 \mathrm{~h}$ and analyzed using a CCK-8 assay. B. Cells were seeded into a 96-well microplate, treated with piperlongumine $(1.5 \mu \mathrm{M})$ for $12 \mathrm{~h}$, and then treated with different concentrations of BTZ for $36 \mathrm{~h}$ and measured using a CCK-8 assay. C. RPMI8226/BTZ100 cells were treated with $0.5,1,2.5$, or $5 \mu \mathrm{M}$ piperlongumine for $24 \mathrm{~h}$, and cleaved caspase-3 and caspase-9 levels were detected. D. RPMI8226/BTZ100 cells were treated with piperlongumine $(4 \mu \mathrm{M})$ for 10, 30, 60, or $120 \mathrm{~min}$, and p-STAT3 and STAT3 levels were measured. E. RPMI8226/BTZ100 cells were exposed to piperlongumine $(2.5 \mu \mathrm{M})$ for $12 \mathrm{~h}$ followed by treatment with BTZ for $24 \mathrm{~h}$, and poly-ubiquitinated protein levels were measured. F. RPMI8226/BTZ100 cells were treated with $0.5,1,2.5$, or $5 \mu \mathrm{M}$ piperlongumine for $24 \mathrm{~h}$, and $\beta 1, \beta 1 \mathrm{i}, \beta 5, \beta 5 \mathrm{i}$, and $\alpha 5$ levels were measured. The data shown are representative of three independent experiments. G. RPMI8226/BTZ100 cells were exposed to piperlongumine $(1,2.5$, or $5 \mu \mathrm{M})$ for $24 \mathrm{~h}$, and proteasome activities were measured. Error bars represent standard deviations of the mean determined in a representative experiment performed in triplicate, and the results are representative of three independent experiments. 
Mechanistic studies have demonstrated that piperlongumineinduced apoptosis is associated with inhibition of the STAT3 signal pathway. Here, piperlongumine markedly decreased the expression of STAT3-regulated genes, such as VEGF, c-myc, Bcl-2, and survivin.

Piperlongumine has strong cytotoxicity in both BTZ-sensitive and BTZ-resistant MM cells, with no significant cross resistance. Our data indicate that this may be due to piperlongumine's ability to downregulate STAT3 signal pathway activity. The JAK-STAT3 signal pathway plays a critical role in the pathophysiology of MM, strongly promoting proliferation, survival, and drug resistance in myeloma cells [30]. The STAT3 protein is composed of 6 functional domains; the SH2 domain and the transactivation domain are especially critical for STAT3 phosphorylation, dimerization, and activation
A

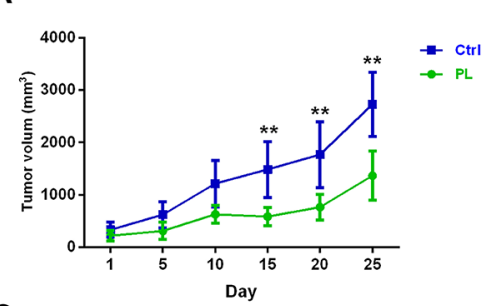

C

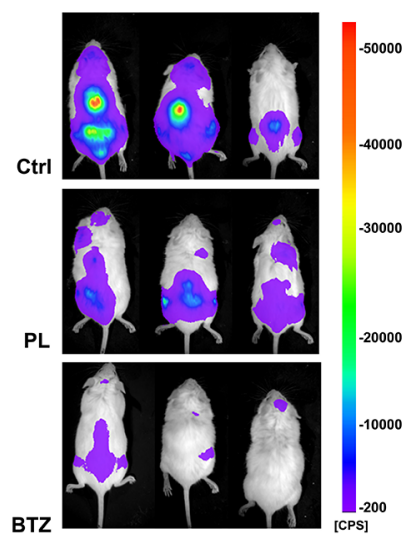

B

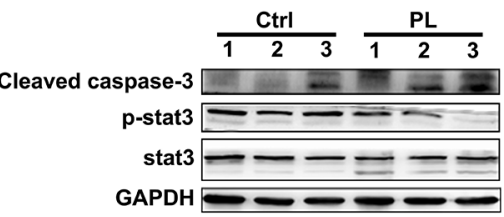

D

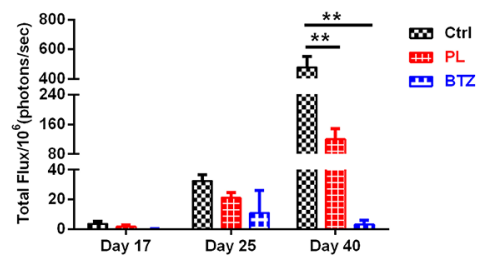

E

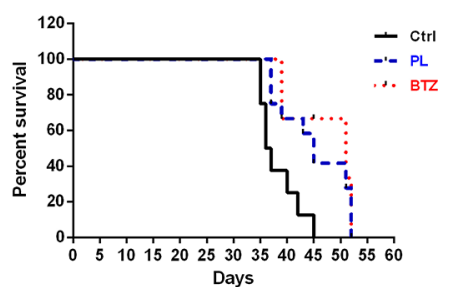

F

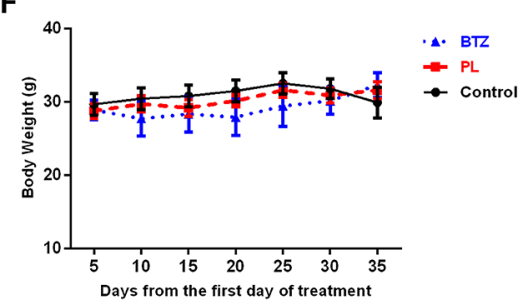

G
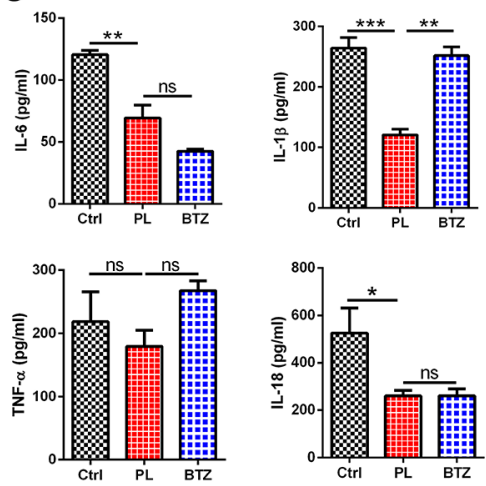

I

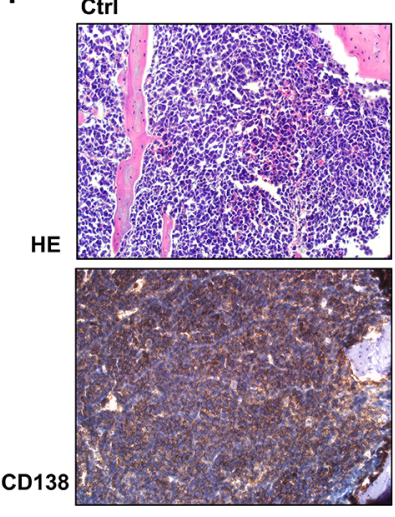

PL

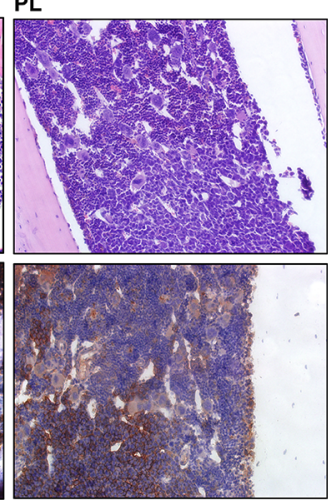

BTZ

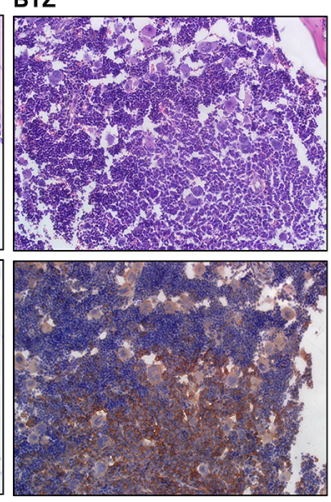

H

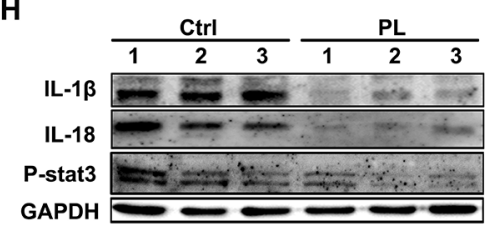

$J$

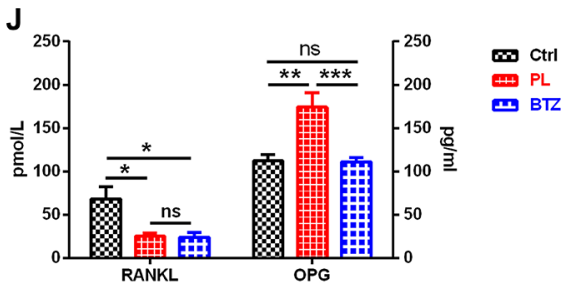

Figure 6: Piperlongumine exerted anti-MM effects and reduced MM-induced bone lesions in a disseminated murine model of human MM. A. Nude mice were treated with vehicle $(\mathrm{n}=6)$ or piperlongumine $(50 \mathrm{mg} / \mathrm{kg}, \mathrm{n}=6)$ for 3 weeks. Piperlongumine treatment inhibited tumor growth compared to the control $(p<0.01)$. B. WB analysis of mouse tumors showed that piperlongumine increased cleaved caspase-3 levels and decreased p-STAT3 levels. C. NSG mice were inoculated intravenously with RPMI8226-Fluc-GFP cells and then treated with vehicle $(\mathrm{n}=8)$, piperlongumine $(50 \mathrm{mg} / \mathrm{kg}, \mathrm{n}=12)$, or bortezomib $(1.5 \mathrm{mg} / \mathrm{kg}, \mathrm{n}=10)$ for 2 weeks. Tumor growth was measured by bioluminescence imaging $(\log$ scale). D. Bioluminescence imaging reported as mean photon flux $( \pm \mathrm{SD})$. E. Log-rank analysis showed that median OS was prolonged in mice treated with piperlongumine (49 days) compared to the control group ( 39.5 days) $(P<0.01)$. F. Treatment with piperlongumine or bortezomib did not significantly affect mouse body weights. G. Levels of secreted IL-6, IL-18, IL$1 \beta$, and TNF- $\alpha$ were measured in bone marrow cell supernatant using an ELISA. H. WB analysis showed that piperlongumine treatment reduced IL- $1 \beta$ and IL-1 8 levels in bone marrow cells compared to the control group. I. The extremities of the mice were stained with H\&E and immunohistochemically analyzed for the indicated proteins. Original magnification, $\times 400$. J. Levels of secreted RANKL and OPG in bone marrow cell supernatants were measured using an ELISA. ${ }^{* *} P<0.001 ; * * P<0.01 ;{ }^{*} P<0.05$. ns: not significant. 
$[23,31]$. Because the Cys712 residue is located near the $\mathrm{SH} 2$ domain, it is the critical site for covalent interactions between STAT3 and drug agents [23]. Here, we demonstrate for the first time that piperlongumine inhibits the STAT3 signal pathway in MM cells by directly binding to the Cys 712 residue; a C712A mutation in STAT3 largely reversed the anti-myeloma effects of piperlongumine. Thus, piperlongumine may exert anti-myeloma effects by specifically inhibiting Cys712-dependent STAT3 activation.

Previous studies have demonstrated that levels of the constitutive proteasome subunit $\beta 5$, which harbors mutations in the BTZ-binding pocket, are increased, and non-mutated immunoproteasome subunit levels are decreased, in BTZ-resistant cell lines [24, 32]. B5i and $\beta 1 \mathrm{i}$ expression are also down-regulated in BTZ-resistant MM cells, which may further contribute to resistance to BTZinduced cytotoxicity. These data suggest that up-regulation of immunoproteasome expression may effectively reduce BTZ resistance in these cells [24]. Interestingly, we found that piperlongumine increased $\beta 5 \mathrm{i}$ expression and facilitated sensitization to BTZ. Furthermore, the immunoproteasome is slightly more efficient than the $20 \mathrm{~S}$ proteasome in recognizing and degrading nascent oxidantdamaged proteins [33]. Recent studies have demonstrated that piperlongumine contributes to cancer cell death by targeting stress responses to ROS [9, 34-36]. Consistent with these studies, we found that piperlongumine increased intracellular ROS levels in MM cells. Therefore, we speculate that piperlongumine-induced oxidative stress may contribute to upregulation of the immunoproteasome $(\beta 5 i)$, which in turn may increase selective degradation of oxidized proteins and help maintain protein homeostasis.

In summary, our results demonstrate that piperlongumine has strong anti-MM activity and inhibits STAT3 activation by binding directly to the Cys 712 residue. Piperlongumine may therefore be a promising new agent for the clinical treatment of multiple myeloma, especially for bortezomib-resistant myeloma.

\section{MATERIALS AND METHODS}

\section{Cell lines}

OPM2, MM1R, U266, and IM-9 human multiple myeloma cell lines were obtained from the American Type Culture Collection (ATCC). The NCI-H929 cell line was obtained from the China Infrastructure of Cell Line Resources. The RPMI-8226 (BTZ IC I0 $=2.6 \pm 0.3$ ), RPMI8226/BTZ7 $\left(\mathrm{IC}_{50}=12.1 \pm 0.7\right.$, resistance factor: $4.5)$, and RPMI8226/BTZ100 $\left(\mathrm{IC}_{50}=105.9 \pm 14.9\right.$, resistance factor: 39.5$)$ cell lines were kindly provided by Dr. J Cloos (VU University Medical Center) [24, 32]. All cells were cultured in RPMI 1640 (Gibco, USA) supplemented with $10 \%$ fetal bovine serum (Gibco, USA). The human stromal cell line HS-5 was also obtained from ATCC.

\section{Antibodies and reagents}

Piperlongumine was purchased from Cayman Chemical (Ann Arbor, Michigan, USA). The apoptosis assay kit was purchased from eBioscience and the cell proliferation ELISA kit (BrdU) was purchased from Roche Diagnostics. Propidium iodide (PI), Ribonuclease A (RNase A), and dimethyl sulfoxide (DMSO) were purchased from Sigma-Aldrich (Shanghai, China). The cell counting kit-8 (CCK-8) was purchased from Dojindo Laboratories (Japan). Antibodies against p-STAT3, STAT3, PSMB6 ( $\beta 1)$, PSMB8 ( $\beta 5 \mathrm{i})$, CDK2, cyclin E2, cyclin A, Bax, Bcl-2, c-myc, p21, p27, survivin, p65, p-p65, IL-1 $\beta$, Histone-H3, caspase- 8 , cleaved caspase- 9 , and cleaved caspase-3 were purchased from Cell Signaling Technology (Beverly, MA, USA). PSMB5 ( $\beta 5$ ) and PSMB9 ( $\beta 11$ ) were purchased from Abcam (Cambridge, MA). p38, p-p38, IL18, ERK, p-ERK, and GAPDH were purchased from Santa Cruz Biotechnology, Inc. (USA). PSMA5 was purchased from Bioworld Technology. CD138 was purchased from Proteintech. Recombinant human macrophage colonystimulating factor (M-CSF) and human soluble receptor activator of NF- $\kappa B$ ligand (sRANK Ligand) were purchased from PeproTech (Rocky Hill, NJ). STAT3CA (constitutively active, A661C, N663C) plasmid was purchased from Addgene. The Cys712 of STAT3-CA was mutated to Ala (STAT3-CA-C712A) using the Fast Mutagenesis System.

\section{Cell proliferation assay}

NCI-H929 cells $\left(2 \times 10^{4}\right.$ cells/well $)$ were seeded into 96-well plates and treated with different concentrations of piperlongumine. After $24 \mathrm{~h}$, cells were labeled with BrdU $(10 \mu \mathrm{M})$ for analysis of cell proliferation according to the manufacturer's protocol.

\section{Caspase activity assay}

Caspase activity was detected using colorimetric assay kits (Bio-Box, China) according to the manufacturer's instructions. Briefly, 100-200 $\mu \mathrm{g}$ of cell lysates were added to a buffer containing a p-nitroaniline (pNA)-conjugated substrates for caspase-3 (Ac-DEVD-pNA), caspase-9 (AcLEHD-pNA), or caspase-8 (Ac-IETD-pNA) for analysis of caspase activity at $405 \mathrm{~nm}$ with a Multiskan FC Microplate Photometer (Thermo Scientific).

\section{Measurement of mitochondrial membrane potential}

The JC-1 (5', 6, 6'-tetrachloro-1, 1', 3, 3'-te traethylbenzimidazolylcarbocyanine iodide) Assay Kit (Life technologies, USA) was used to measure mitochondrial membrane potential disruption according to the manufacturer's protocol. Positive control cells were incubated with CCCP (carbonyl cyanide 
3-chlorophenylhydrazone, $50 \mathrm{mM}$ final concentration) for 5 min. Cells were washed once with PBS and analyzed using a flow cytometer.

\section{Measurement of pro-inflammatory cytokines}

HS-5 $\left(2 \times 10^{5}\right)$ cells were seeded into 24 -well plates for $24 \mathrm{~h}$ to establish bone marrow stromal layers. NCI-H929 cells $\left(2 \times 10^{5}\right)$ were added to the wells either alone or with the HS-5 stromal cell layer. Conditioned media was then harvested after $48 \mathrm{~h}$ of co-culture and secreted VEGF levels were detected using an enzyme-linked immunosorbent assay (ELISA) (MultiSciences). IL-1 $\beta$, IL-18, IL-6, TNF- $\alpha$, RANKL, and OPG levels in bone marrow cell supernatants were also measured by ELISA. The minimum detectable level for the cytokines was $10.0 \mathrm{pg} / \mathrm{mL}$. All measurements were performed in triplicate.

\section{In vitro osteoclast culture and activity assay}

Osteoclast (OC) differentiation from healthy donor peripheral blood mononuclear cells (PBMCs) was performed as previously described [37], [38]. The culture medium containing $25 \mathrm{ng} / \mathrm{mL}$ of M-CSF and $50 \mathrm{ng} / \mathrm{mL}$ of RANKL was refreshed three times a week, and piperlongumine was added at 2 or $4 \mu \mathrm{M}$. After 15 days of culture, cells were fixed and stained using a TRACP\&ALP double-stain kit (TaKaRa Company) according to the manufacturer's instructions. TRACP + OCs contain three or more nuclei per cell, and each OC formation assay was performed at least three times using PBMCs from different donors.

\section{Intact cell-based chymotrypsin-like, trypsin-like, and caspase-like proteasome activities}

An intact cell-based Proteasome-Glo assay kit (Promega, Madison, WI) was used to measure basal and piperlongumine-induced proteasome activity according to the manufacturer's instructions. MM cells were exposed to piperlongumine for $24 \mathrm{~h}$, and 20,000 cells were collected from each well for detection. After $10 \mathrm{~min}$ of incubation with an equal volume of Proteasome-Glo cell based reagent, luminescence was measured with a GloMAX 96 Microplate Luminometer (Promega).

\section{MM xenograft mouse model (plasmacytoma model)}

To evaluate the anti-MM activity of piperlongumine in vivo, $5 \times 10^{6}$ NCI-H929 cells were injected subcutaneously into the right flanks of nude mice (5-6 weeks old, female, Shanghai Slac Laboratory Animal Co. Ltd., Shanghai) [39]. Once tumors were measurable, mice received intraperitoneal injections of $50 \mathrm{mg} / \mathrm{kg}$ piperlongumine in 10\% DMSO and 10\% Tween 80 in water on 5 consecutive days a week for 3 weeks. The control group received vehicle injections under the same schedule. Tumor lengths and widths were measured every five days using calipers, and tumor volume was calculated with the formula $\mathrm{V}=\pi / 6\left(\mathrm{a} \times \mathrm{b}^{2}\right)$, where $\mathrm{a}$ and $\mathrm{b}$ are longer and shorter tumor diameters, respectively. Mice were killed when tumors reached $2 \mathrm{~cm}^{3}$ or became ulcerated. Survival and tumor growth were evaluated from the first day of treatment until death. Tissue samples were collected, minced, and homogenized to extract whole cell lysates, and the clarified supernatants were used for Western blotting analyses.

\section{Disseminated MM model}

To induce disseminated MM, male NOD-scid IL2rynull (NSG) mice (Beijing Vitalstar Biotechnology Co., Ltd.) were inoculated intravenously with $5 \times 10^{6}$ RPMI-8226-Fluc-GFP cells in $250 \mu \mathrm{L}$ PBS and imaged five days later to determine baseline bioluminescence. Mice were divided into three groups with similar mean bioluminescence and received either 5 consecutive IP doses of vehicle (days $1-5$ ), $50 \mathrm{mg} / \mathrm{kg}$ piperlongumine (days $1-5$ ), or $1.5 \mathrm{mg} / \mathrm{kg}$ bortezomib IP twice weekly (days 1 and 5), for 2 consecutive weeks. Bioluminescence imaging was performed weekly to monitor disease progression, and body weights were measured every 5 days.

\section{Bone marrow H\&E and immunohistochemistry staining}

Mice were sacrificed and femurs and tibia were isolated bilaterally, fixed with $4 \%$ paraformaldehyde solution for $48 \mathrm{~h}$, decalcified with $10 \%$ EDTA for another $48 \mathrm{~h}$, dehydrated, waxed, and sliced into 4 $\mu \mathrm{m}$ sections with an RM2126 microtome. After H\&E staining, pathologic changes were evaluated using a light microscope. For immunohistochemistry, CD138 (1:100 dilution) antibody was added followed by incubation with HRP-conjugated secondary antibody. Sections were then prepared for and viewed using fluorescent microscopy.

\section{Statistical analysis}

Data are shown as means \pm SD or SE. Statistical significance between 2 treatment groups was analyzed using unpaired Student'st-tests with 2-tailed $P$ values; statistical significance between multiple treatment groups was analyzed using one-way ANOVAs; statistical significance between multiple treatment groups over time was analyzed using 2-way ANOVAs; overall survival (OS) was measured using Kaplan-Meier survival analysis. All statistical analyses were conducted using GraphPad Prism software (version 6.0). $P<0.05$ was considered statistically significant. 


\section{Abbreviations}

BTZ, bortezomib; PL, Piperlongumine; MM, multiple myeloma; BM, bone marrow; CDK, cyclindependent kinase; STAT3, signal transducer and activator of transcription 3; UPS, ubiquitin-proteasome system; ROS, reactive oxygen species; DMSO, dimethyl sulfoxide; ELISA, enzyme-linked immunosorbent assay; IFN $\gamma$, interferon $\gamma$; M-CSF, macrophage colonystimulating factor; OC, osteoclast cell; sRANK, soluble receptor activator of $\mathrm{NF \kappa B}$; VEGF, vascular endothelial growth factor.

\section{ACKNOWLEDGMENTS}

This work was supported by the National Natural Science Foundation of China (81302034, 81400167 and 81670142); the Foundation of Jiangsu Province Six Talents Peak (2015-WSW-057); Natural Science Foundation of Jiangsu Province (BK20140227).

\section{CONFLICTS OF INTEREST}

The authors have no conflict of interest to declare.

\section{REFERENCES}

1. Dimopoulos MA, San-Miguel JF and Anderson KC. Emerging therapies for the treatment of relapsed or refractory multiple myeloma. Eur J Haematol. 2011; 86:1-15.

2. Kaufman JL, Fabre C, Lonial S and Richardson PG. Histone deacetylase inhibitors in multiple myeloma: rationale and evidence for their use in combination therapy. Clin Lymphoma Myeloma Leuk. 2013; 13:370-376.

3. Ciechanover A, Orian A and Schwartz AL. Ubiquitinmediated proteolysis: biological regulation via destruction. Bioessays. 2000; 22:442-451.

4. Cavo M. Proteasome inhibitor bortezomib for the treatment of multiple myeloma. Leukemia. 2006; 20:1341-1352.

5. Almond JB and Cohen GM. The proteasome: a novel target for cancer chemotherapy. Leukemia. 2002; 16:433-443.

6. Krishnan SR, Jaiswal R, Brown RD, Luk F and Bebawy $\mathrm{M}$. Multiple myeloma and persistence of drug resistance in the age of novel drugs (Review). International journal of oncology. 2016; 49:33-50.

7. Park BS, Son DJ, Choi WS, Takeoka GR, Han SO, Kim TW and Lee SE. Antiplatelet activities of newly synthesized derivatives of piperlongumine. Phytotherapy research. 2008; 22:1195-1199.

8. Seo YH, Kim JK and Jun JG. Synthesis and biological evaluation of piperlongumine derivatives as potent antiinflammatory agents. Bioorganic \& medicinal chemistry letters. 2014; 24:5727-5730.
9. Han SS, Han S and Kamberos NL. Piperlongumine inhibits the proliferation and survival of B-cell acute lymphoblastic leukemia cell lines irrespective of glucocorticoid resistance. Biochemical and biophysical research communications. 2014; 452:669-675.

10. Jin HO, Lee YH, Park JA, Lee HN, Kim JH, Kim JY, Kim B, Hong SE, Kim HA, Kim EK, Noh WC, Kim JI, Chang YH, Hong SI, Hong YJ, Park IC, et al. Piperlongumine induces cell death through ROS-mediated CHOP activation and potentiates TRAIL-induced cell death in breast cancer cells. J Cancer Res Clin Oncol. 2014; 140:2039-2046.

11. Liu QR, Liu JM, Chen Y, Xie XQ, Xiong XX, Qiu XY, Pan F, Liu D, Yu SB and Chen XQ. Piperlongumine inhibits migration of glioblastoma cells via activation of ROSdependent p38 and JNK signaling pathways. Oxidative medicine and cellular longevity. 2014; 2014:653732.

12. Ryu J, Kim MJ, Kim TO, Huh TL and Lee SE. Piperlongumine as a potential activator of AMP-activated protein kinase in HepG2 cells. Nat Prod Res. 2014; 28:2040-2043.

13. Shrivastava S, Kulkarni P, Thummuri D, Jeengar MK, Naidu VG, Alvala M, Redddy GB and Ramakrishna S. Piperlongumine, an alkaloid causes inhibition of PI3 K/ $\mathrm{Akt} / \mathrm{mTOR}$ signaling axis to induce caspase-dependent apoptosis in human triple-negative breast cancer cells. Apoptosis. 2014; 19:1148-1164.

14. Bharadwaj U, Eckols TK, Kolosov M, Kasembeli MM, Adam A, Torres D, Zhang X, Dobrolecki LE, Wei W, Lewis MT, Dave B, Chang JC, Landis MD, Creighton CJ, Mancini MA and Tweardy DJ. Drug-repositioning screening identified piperlongumine as a direct STAT3 inhibitor with potent activity against breast cancer. Oncogene. 2014; 0.

15. Makhov P, Golovine K, Teper E, Kutikov A, Mehrazin R, Corcoran A, Tulin A, Uzzo RG and Kolenko VM. Piperlongumine promotes autophagy via inhibition of Akt/ mTOR signalling and mediates cancer cell death. Br J Cancer. 2014; 110:899-907.

16. Ginzburg S, Golovine KV, Makhov PB, Uzzo RG, Kutikov A and Kolenko VM. Piperlongumine inhibits NF-kappaB activity and attenuates aggressive growth characteristics of prostate cancer cells. Prostate. 2014; 74:177-186.

17. Wang Y, Wang JW, Xiao X, Shan Y, Xue B, Jiang G, He Q, Chen J, Xu HG, Zhao RX, Werle KD, Cui R, Liang J, Li YL and $\mathrm{Xu}$ ZX. Piperlongumine induces autophagy by targeting p38 signaling. Cell death \& disease. 2013; 4:e824.

18. Jarvius M, Fryknas M, D'Arcy P, Sun C, Rickardson L, Gullbo J, Haglund C, Nygren P, Linder S and Larsson R. Piperlongumine induces inhibition of the ubiquitinproteasome system in cancer cells. Biochemical and biophysical research communications. 2013; 431:117-123.

19. Manier S, Sacco A, Leleu X, Ghobrial IM and Roccaro AM. Bone marrow microenvironment in multiple myeloma progression. J Biomed Biotechnol. 2012; 2012:157496. 
20. Kawano Y, Moschetta M, Manier S, Glavey S, Gorgun GT, Roccaro AM, Anderson KC and Ghobrial IM. Targeting the bone marrow microenvironment in multiple myeloma. Immunol Rev. 2015; 263:160-172.

21. Ding JH, Yuan LY, Huang RB and Chen GA. Aspirin inhibits proliferation and induces apoptosis of multiple myeloma cells through regulation of $\mathrm{Bcl}-2$ and $\mathrm{Bax}$ and suppression of VEGF. Eur J Haematol. 2014; 93:329-339.

22. Yang Y, Blair HC, Shapiro IM and Wang B. The Proteasome Inhibitor Carfilzomib Suppresses Parathyroid Hormoneinduced Osteoclastogenesis through a RANKL-mediated Signaling Pathway. The Journal of biological chemistry. 2015 .

23. Yu X, He L, Cao P and Yu Q. Eriocalyxin B Inhibits STAT3 Signaling by Covalently Targeting STAT3 and Blocking Phosphorylation and Activation of STAT3. PLoS ONE. 2015; 10:e128406.

24. Niewerth D, Kaspers GJ, Assaraf YG, van Meerloo J, Kirk CJ, Anderl J, Blank JL, van de Ven PM, Zweegman S, Jansen G and Cloos J. Interferon-gammainduced upregulation of immunoproteasome subunit assembly overcomes bortezomib resistance in human hematological cell lines. Journal of hematology \& oncology. 2014; 7:7.

25. Han K, Xu X, Chen G, Zeng Y, Zhu J, Du X, Zhang Z, Cao $\mathrm{B}$, Liu $\mathrm{Z}$ and Mao X. Identification of a promising PI3K inhibitor for the treatment of multiple myeloma through the structural optimization. Journal of hematology \& oncology. 2014; 7:9.

26. Garcia-Gomez A, Sanchez-Guijo F, Del Canizo MC, San Miguel JF and Garayoa M. Multiple myeloma mesenchymal stromal cells: Contribution to myeloma bone disease and therapeutics. World J Stem Cells. 2014; 6:322-343.

27. Sezer O, Heider U, Zavrski I, Kuhne CA and Hofbauer LC. RANK ligand and osteoprotegerin in myeloma bone disease. Blood. 2003; 101:2094-2098.

28. Zhang S, Suvannasankha A, Crean CD, White VL, Chen CS and Farag SS. The novel histone deacetylase inhibitor, AR-42, inhibits gp130/Stat3 pathway and induces apoptosis and cell cycle arrest in multiple myeloma cells. International journal of cancer. 2011; 129:204-213.

29. Sharkey J, Khong T and Spencer A. PKC412 demonstrates JNK-dependent activity against human multiple myeloma cells. Blood. 2007; 109:1712-1719.

30. Scuto A, Krejci P, Popplewell L, Wu J, Wang Y, Kujawski M, Kowolik C, Xin H, Chen L, Kretzner L, Yu H, Wilcox WR, Yen Y, Forman S and Jove R. The novel JAK inhibitor
AZD1480 blocks STAT3 and FGFR3 signaling, resulting in suppression of human myeloma cell growth and survival. Leukemia. 2011; 25:538-550.

31. Becker S, Groner B and Muller CW. Three-dimensional structure of the Stat3beta homodimer bound to DNA. Nature. 1998; 394:145-151.

32. Franke NE, Niewerth D, Assaraf YG, van Meerloo J, Vojtekova K, van Zantwijk CH, Zweegman S, Chan ET, Kirk CJ, Geerke DP, Schimmer AD, Kaspers GJ, Jansen G and Cloos J. Impaired bortezomib binding to mutant beta5 subunit of the proteasome is the underlying basis for bortezomib resistance in leukemia cells. Leukemia. 2012; 26:757-768.

33. Pickering AM, Koop AL, Teoh CY, Ermak G, Grune T and Davies KJ. The immunoproteasome, the 20S proteasome and the PA28alphabeta proteasome regulator are oxidativestress-adaptive proteolytic complexes. The Biochemical journal. 2010; 432:585-594.

34. Raj L, Ide T, Gurkar AU, Foley M, Schenone M, Li X, Tolliday NJ, Golub TR, Carr SA, Shamji AF, Stern AM, Mandinova A, Schreiber SL and Lee SW. Selective killing of cancer cells by a small molecule targeting the stress response to ROS. Nature. 2011; 475:231-234.

35. Xiong XX, Liu JM, Qiu XY, Pan F, Yu SB and Chen XQ. Piperlongumine induces apoptotic and autophagic death of the primary myeloid leukemia cells from patients via activation of ROS-p38/JNK pathways. Acta pharmacologica Sinica. 2015; 36:362-374.

36. Chen Y, Liu JM, Xiong XX, Qiu XY, Pan F, Liu D, Lan SJ, Jin S, Yu SB and Chen XQ. Piperlongumine selectively kills hepatocellular carcinoma cells and preferentially inhibits their invasion via ROS-ER-MAPKs-CHOP. Oncotarget. 2015; 6:6406-6421. doi: 10.18632/oncotarget.3444.

37. Yao Y, Xia D, Bian Y, Sun Y, Zhu F, Pan B, Niu M, Zhao K, $\mathrm{Wu}$ Q, Qiao J, Fu C, Li Z and Xu K. Alantolactone induces G1 phase arrest and apoptosis of multiple myeloma cells and overcomes bortezomib resistance. Apoptosis. 2015; 20:1122-1133.

38. Vallet S, Raje N, Ishitsuka K, Hideshima T, Podar K, Chhetri S, Pozzi S, Breitkreutz I, Kiziltepe T, Yasui H, Ocio EM, Shiraishi N, Jin J, Okawa Y, Ikeda H, Mukherjee $\mathrm{S}$, et al. MLN3897, a novel CCR1 inhibitor, impairs osteoclastogenesis and inhibits the interaction of multiple myeloma cells and osteoclasts. Blood. 2007; 110:3744-3752.

39. Yao Y, Luo J, Bian Y, Sun Y, Shi M, Xia D, Niu M, Zhao $\mathrm{K}$, Zeng L, Chen W, Li Z and Xu K. Sprouty2 regulates proliferation and survival of multiple myeloma by inhibiting activation of the ERK1/2 pathway in vitro and in vivo. Exp Hematol. 2016; 44:474-482 e472. 